\title{
KMT2A/C mutations function as a potential predictive biomarker for immunotherapy in solid tumors
}

\author{
Rui Zhang ${ }^{1 \dagger}$, Hao-Xiang $\mathrm{Wu}^{2 \dagger}$, Ming $\mathrm{Xu}^{1+}$ and Xiaoyan $\mathrm{Xie}^{1 *}$
}

\begin{abstract}
Epigenetic factors play important roles in tumor immunology. Histone-lysine N-methyltransferase 2 (KMT2) family genes exert histone $\mathrm{H} 3$ methylation, but its role in immunotherapy remains unclear. Our study is the first to investigate the correlation between KMT2 gene mutations and the clinical benefit of immune checkpoint inhibitors (ICI) treatment. We firstly collected a primary ICl-treated cohort $(n=546)$ and found that patients with KMT2A/C mutations yielded better prognosis in terms of progression-free survival (PFS, Hazard ratio $[H R]=0.66, P=0.002$ ), objective response rate (ORR, $40.9 \%$ vs $20.3 \%, P<0.001)$, durable clinical benefit (DCB, $48.3 \%$ vs $29.8 \%, P=0.001$ ) and overall survival $(O S, H R=0.70, P=0.033)$. Furthermore, we validated the predictive potential of $K M T 2 A / C$ mutations in an expanded ICl-treated cohort $(n=1395)$. KMT2A/C-mutant patients achieved better OS compared with KMT2A/C-wildtype patients ( $H R=0.68, P=0.003)$; and the survival advantages appeared in the majority of cancer subtypes. Our study suggests that KMT2A/C mutations function as a novel and potential predictive biomarker for $\mathrm{ICl}$ treatment in multiple solid tumors and the underlying mechanism is worth investigating.
\end{abstract}

Keywords: Biomarker, Immune checkpoint inhibitors, KMT2A/C, Pan-cancer analysis

\section{To the Editor,}

Recent years have witnessed the great success of immune checkpoint inhibitors (ICIs) in treating multiple advanced tumors [1]. However, clinical response of ICIs varies and identification of predictive biomarkers is still in urgent demand.

Growing evidence suggests that epigenetic factors play important roles in immuno-oncology [2, 3]. For example, the mutation in DNA demethylase TET1 predicted higher response rate in ICI-treated patients [4], and DNA methyltransferase inhibitors (DNMTi) and histone deacetylase inhibitors (HDACi) showed promising potentials to augment the efficacy of ICIs $[5,6]$.

\section{* Correspondence: xiexyan@mail.sysu.edu.cn}

${ }^{\dagger}$ Rui Zhang, Hao-Xiang Wu and Ming Xu contributed equally to this work. 'Department of Medical Ultrasound, Division of Interventional Ultrasound, The First Affiliated Hospital, Sun Yat-sen University, No. 58 Zhongshan 2nd Road, Guangzhou 510080, China

Full list of author information is available at the end of the article
The KMT2 family genes, one of the important epigenetic regulator genes, were initially recognized in mixedlineage leukemia (MLL) caused by the rearrangement of KMT2A on chromosome 11q23 [7, 8]. Recent exomesequencing studies revealed that KMT2 genes were among the most frequently mutated genes in various types of human cancers [9]. The KMT2 proteins, namely KMT2A, KMT2B, KMT2C and KMT2D, function as methylating histone $\mathrm{H} 3$ on lysine 4 (H3K4) to promote genome accessibility and transcription, but its role in immunotherapy remains unclear. Herein, we investigated the correlation between KMT2 gene mutations and clinical benefit of ICI treatment in the human pancancer setting, which was the first time to the best of our knowledge.

To address this issue, we collected a primary ICItreated cohort $(n=546)$ with annotated response and mutational data as well as survival data from seven 
published studies (see Methods), composing of bladder cancer, esophagogastric cancer, head and neck cancer, hepatocellular carcinoma, melanoma and non-small-cell lung cancer (baseline characteristics were shown in Table S1). We found that both KMT2A $(P=0.007)$ and KMT2C $(P=0.041)$ mutations significantly correlated with better progression-free survival (PFS) in patients receiving ICI treatment, while KMT2B $(P=0.964)$ and KMT2D $(P=0.200)$ did not. Then we combined KMT2A and $\mathrm{KMT} 2 \mathrm{C}$ mutation as $\mathrm{KMT} 2 \mathrm{~A} / \mathrm{C}$ mutations, and found that patients harboring $\mathrm{KMT} 2 \mathrm{~A} / \mathrm{C}$ mutations correlated with longer PFS most significantly (Fig. 1a, P =
0.002). Moreover, the Bonferroni-corrected $p$ value of the association between KMT2A/C-mutant and PFS was 0.010 , indicating that $\mathrm{KMT} 2 \mathrm{~A} / \mathrm{C}$-mutant was robustly associated with improved PFS in patients treated with ICIs.

We subsequently analyzed the association between KMT2A/C mutations and objective response rate (ORR), durable clinical benefit (DCB) and overall survival (OS) in patients receiving ICI treatment. Compared with KMT2A/C-wildtype patients, KMT2A/C-mutant patients achieved significantly higher ORR $(40.9 \%$ vs. $20.3 \%, P<0.001$; Fig. 1b, left panel), better DCB $(48.3 \%$

A

\begin{tabular}{|c|c|c|c|c|c|c|c|c|}
\hline \multirow{2}{*}{ Gene } & \multicolumn{2}{|c|}{ Mut group } & \multicolumn{2}{|c|}{ Wt group } & \multirow{2}{*}{ Favors Mut } & \multirow{2}{*}{ Favors Wt } & \multirow{2}{*}{ HR $(95 \% \mathrm{Cl})$} & \multirow{2}{*}{$P$} \\
\hline & Events/n & $\begin{array}{l}\text { Median PFS, mo } \\
\quad(95 \% \mathrm{Cl})\end{array}$ & Events/n & $\begin{array}{c}\text { Median PFS, mo } \\
\quad(95 \% \mathrm{Cl})\end{array}$ & & & & \\
\hline КМТ2А & $26 / 43$ & 11.7 (3.49 to NE) & $410 / 503$ & 3.50 (3.06 to 4.05$)$ & $\longrightarrow$ & & 0.57 (0.38 to 0.86$)$ & 0.007 \\
\hline КМТ2B & $14 / 17$ & 4.14 (2.80 to NE) & $191 / 256$ & 4.05 (3.45 to 5.30$)$ & & & 1.00 (0.56 to 1.74$)$ & 0.964 \\
\hline КМТ2C & $46 / 65$ & 5.59 (3.49 to 15.36$)$ & $390 / 481$ & 3.50 (3.03 to 4.05$)$ & $\longrightarrow$ & & 0.72 (0.53 to 0.98$)$ & 0.041 \\
\hline KMT2D & $46 / 61$ & $5.59(4.05$ to 13.17$)$ & $390 / 485$ & 3.45 (2.96 to 3.95$)$ & $\longrightarrow$ & & 0.81 (0.60 to 1.12$)$ & 0.200 \\
\hline КMT2A/C & $63 / 93$ & 5.99 (3.91 to 14.51$)$ & $373 / 453$ & 3.43 (2.93 to 3.95 ) & $\rightarrow-$ & & 0.66 (0.50 to 0.86$)$ & 0.002 \\
\hline & & & & & & 2.0 & & \\
\hline
\end{tabular}

B

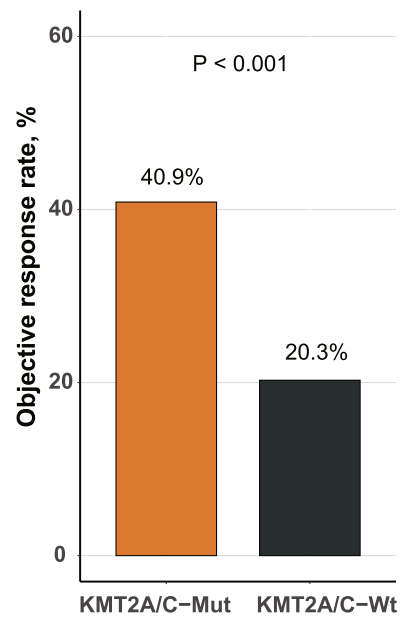

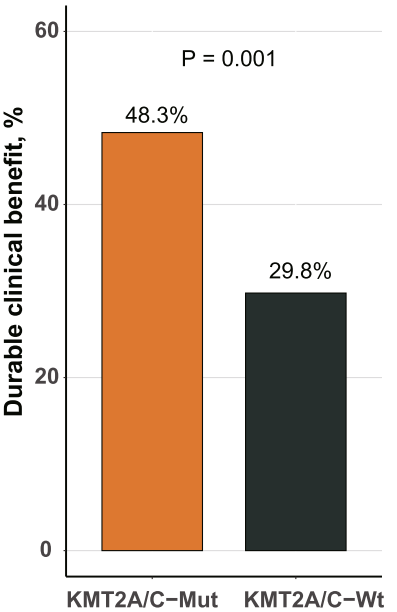

C

Fig. $1 \mathrm{KMT} 2 \mathrm{~A} / \mathrm{C}$ mutations predicted improved clinical outcomes in patients receiving ICI treatment. a The primary ICl-treated cohort $(n=546)$ was divided into the mutant (Mut) group and the wildtype (Wt) group according to the mutation status of individual KMT2 genes and KMT2A/C genes, then the difference of progression-free survival (PFS) between the two groups was compared. $\mathbf{b}$ Histogram demonstrating the proportions of patients achieving objective response (ORR, left panel) and durable clinical benefit (DCB, right panel) in the KMT2A/C-Mut and the KMT2A/CWt groups. c Kaplan-Meier curves of overall survival (OS) according to KMT2A/C mutation status in the ICI-treated cohort ( $n=517$ due to the lacking of OS information of 29 patients). Median OS and 2-year OS were also depicted 
vs. $29.8 \%, P=0.001$; Fig. $1 \mathrm{~b}$, right panel) and improved OS (HR $=0.70$ [95\% confidence interval (CI), 0.51-0.97], $P=0.033$; Fig. 1c). Thus, our findings suggest that KMT2A/C mutations may function as a potential predictive biomarker for ICI treatment.

To further validate the predictive value of KMT2A/ $\mathrm{C}$ mutations, we employed an expanded ICI-treated cohort $(n=1395)$ from Memorial Sloan Kettering Cancer Center on the basis of Samstein and his colleagues' work (see Methods). As shown in Fig. 2a, KMT2A/C-mutant patients achieved significant improved OS (median OS, 41 months [95\% CI, 34-NE]) compared with KMT2A/C-wildtype patients (median OS, 17 months [95\% CI, 15-21]). In order to investigate whether the improvement of OS in KMT2A/Cmutant patients alters with cancer category, we analyzed OS between KMT2A/C-mutant and KMT2A/Cwildtype in different cancer types. And the favorable clinical outcomes for KMT2A/C-mutant were yielded in the majority of the examined cancer types, the overall HR was 0.68 [95\% CI, 0.53-0.88] $(P=0.003$, Fig. 2b), indicating a $32 \%$ lower risk of death. In addition, no survival difference was observed between KMT2A/C-mutant patients and KMT2A/C-wildtype patients in a non-ICI-treated cohort (Figure S1, $n=$ 2252, HR $=0.90$ [95\% CI, 0.71-1.14], $P=0.396$ ), confirming that the improvement of OS after ICI treatment in KMT2A/C-mutant patients compared with that in KMT2A/C-wildtype patients was indeed transformed from its higher response rate, not contributed by its general prognostic impact.
The average occurrence frequency of KMT2A/C-mutant was $17.0 \%$ and $15.3 \%$ in the primary ICI-treated cohort and the expanded ICI-treated cohort, respectively. In addition, we employed the TCGA dataset to investigate the mutational frequency of KMT2A/C in different cancer types. And the results showed that (Figure S2), the mutational frequency of KMT2A/C ranged from $0 \%$ (uveal melanoma) to $30.9 \%$ (skin cutaneous melanoma) among 33 cancer types (average, 12.2\%), which would help in identifying a substantial proportion of cancer patients who would benefit for ICI treatment.

KMT2 genes encoding histone-lysine $\mathrm{N}$-methyltransferase 2 proteins act as writer of histone methylation at important regulatory regions in the genome and thereby impart crucial functions through modulating chromatin structures and DNA accessibility. It's reported that the majority of KMT2A/ $\mathrm{C}$ mutations can cause loss of function in the proteins, which would damage its writer function and lead to changes in the transcriptional regulation [10]. Although there is now no direct evidence between KMT2A/C mutations and tumor immunity, we found that KMT2A/C-mutant tumors tend to have higher tumor mutational burden (TMB) and better clinical outcomes in patients treated with ICI compared with KMT2A/C-wildtype tumors (Figure S3), which suggested that KMT2A/C-mutant may sensitize tumors to ICI treatment at least partially by increasing tumor immunogenicity. As TMB-high has already been established as solid predictive biomarker for ICI treatment, we further evaluated the predictive function of KMT2A/C-mutant in TMB-low patients and found that KMT2A/C-mutant could still be predictive of better ORR $(32.6 \%$ vs $17.9 \%, P=0.029)$ in the TMB-low

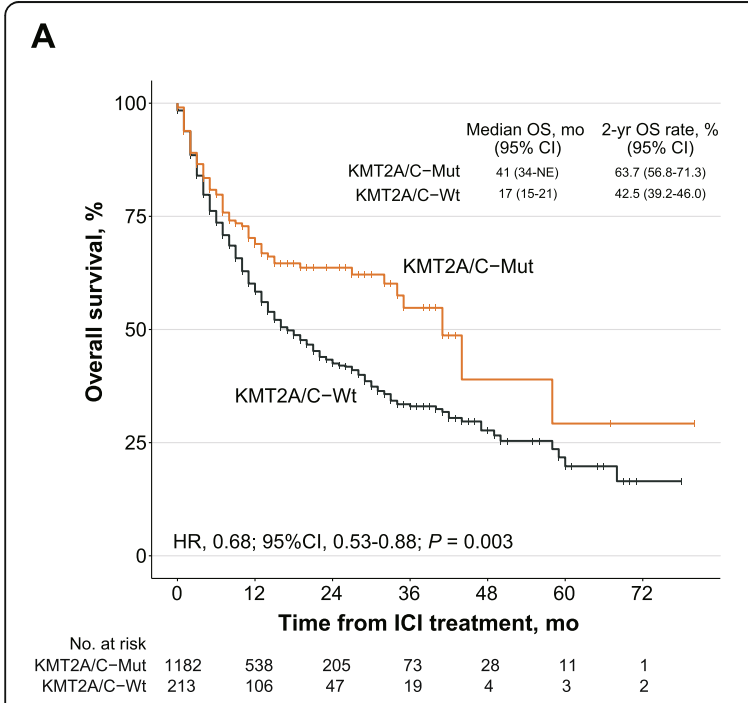

B

\begin{tabular}{|c|c|c|c|c|c|}
\hline Cancer type & Cases & $\operatorname{HR}(95 \% \mathrm{CI})$ & Favors Mut & Favors Wt & $P$ \\
\hline Bladder cancer & 211 & $0.69(0.42$ to 1.15$)$ & & & 0.154 \\
\hline Breast Cancer & 44 & $0.32(0.04$ to 2.39$)$ & & & 0.266 \\
\hline Colorectal Cancer & 109 & $0.42(0.18$ to 0.98$)$ & & & 0.046 \\
\hline Esophagogastric Cancer & 86 & 0.61 (0.08 to 4.63$)$ & & & 0.631 \\
\hline Glioma & 116 & $1.41(0.33$ to 5.99$)$ & & & 0.641 \\
\hline Head and Neck Cancer & 136 & 0.87 (0.41 to 1.83$)$ & & & 0.708 \\
\hline Melanoma & 315 & $0.64(0.39$ to 1.06$)$ & & & 0.082 \\
\hline Non-Small Cell Lung Cancer & 142 & $1.15(0.58$ to 2.31$)$ & & & 0.687 \\
\hline Renal Cell Carcinoma & 151 & $0.73(0.18$ to 3.03$)$ & & & 0.667 \\
\hline Cancer of Unknown Primary & 85 & $0.44(0.13$ to 1.50$)$ & - & - & 0.188 \\
\hline Overall & 1395 & $0.68(0.53$ to 0.88$)$ & $\rightarrow$ & & 0.003 \\
\hline & & & 0.10 & $\begin{array}{c}1 \\
10.0\end{array}$ & \\
\hline
\end{tabular}

Fig. 2 Validation of the predictive function of KMT2A/C mutations in the expanded ICl-treated cohort. a. Kaplan-Meier curves comparing the overall survival (OS) between the KMT2A/C-Mut group and the KMT2A/C-Wt group in the expanded ICl-treated cohort ( $n=1395$ ). mo, months; yr, years. Median OS and 2-year OS were also depicted. b. Forest plot depicting the OS benefit of KMT2A/C-Mut patients compared with KMT2A/CWt patients by cancer category. Bars represent the $95 \% \mathrm{Cl}$ 
subgroup (Figure S4), suggesting the further predictive value of KMT2A/C-mutant beyond TMB and that other possible mechanisms besides higher immunogenicity could also contribute to the sensitivity of KMT2A/C-mutant to ICI treatment, which warranted further investigation.

This study also has several limitations. Firstly, no available information of PD-L1 expression could be included in our study for comparison, though PD-L1 expression seems to be an "imprecise" predictor of response to ICI treatment $[11,12]$. Secondly, although more than 10 cancer types with ICI data were involved in our analysis, more data are needed concerning the potential predictive function of KMT2A/C-mutant in other cancer types. Our study should be considered as generators of hypotheses, and the best way to confirm this hypothesis is a biomaker-driven and tumor histology-agnostic clinical trial incorporating $\mathrm{KMT} 2 \mathrm{~A} / \mathrm{C}$-mutant as biomarker in patients receiving ICI treatment.

In conclusion, our study is the first to reveal the correlation between KMT2A/C mutations and ICI treatment efficacy, which suggest that KMT2A/C mutations can function as a novel and potential biomarker in multiple solid tumors, and adds great value in the identification of patients who might benefit from ICI treatment and may guide the clinical decision-making on the use of ICI. Further studies on the underlying molecular mechanism that KMT2A/C mutations sensitize patients to ICI treatment are still warranted.

\section{Supplementary Information}

The online version contains supplementary material available at https://doi. org/10.1186/s40364-020-00241-0.

\section{Additional file 1. Methods.}

Additional file 2: Table S1. Patient characteristics between KMT2A/CWt and KMT2A/C-Mut subgroups of the primary ICl-treated cohort. ( $n=$ 546).

Additional file 3: Figure S1. Kaplan-Meier curves comparing the overall survival (OS) between the KMT2A/C-Mut group and the KMT2A/C-Wt group in the non-ICl-treated cohort $(n=2252)$.

Additional file 4: Figure S2. The mutational frequency of KMT2A/C across 33 cancer types in the TCGA cohort.

Additional file 5: Figure S3. Boxplot comparing the tumor mutational burden (TMB) between KMT2A/C-Mut tumors and the KMT2A/C-Wt tumors in the primary $\mathrm{ICl}$-treated cohort.

Additional file 6: Figure S4. Barplot comparing the objective response rate (ORR) between KMT2A/C-Mut patients and the KMT2A/C-Wt patients in the TMB-low subgroup of the primary $\mathrm{ICl}$-treated cohort.

\section{Abbreviations}

Cl: Confidence interval; DCB: Durable clinical benefit; DNMTi: DNA methyltransferase inhibitor; HDACi: Histone deacetylase inhibitors; HR: Hazard ratio; ICl: Immune checkpoint inhibitors; MLL: Mixed-lineage leukemia; H3K4: Histone H3 on lysine 4; KMT2: Histone-lysine N-methyltransferase 2; Mut: Mutant; NE: Not evaluable; ORR: Objective response rate; OS: Overall survival; PFS: Progression-free survival; TMB: Tumor mutational burden; Wt: Wildtype

\section{Acknowledgments}

We would like to thank cBioPortal for publicly available research data and the authors for uploading their data for scientific researches.

\section{Authors' contributions}

All authors participated in study concept and design. RZ and HW dealt with data acquisition, arrangement and analysis. All authors participated in drafting the manuscript. XX and MX revised the manuscript for important intellectual content. The author(s) read and approved the final manuscript.

\section{Funding}

This work was supported by grants from the National Natural Science Foundation of China. (No. 81530055 and No.81501489).

\section{Availability of data and materials}

All of the data used in this study were publicly available in cBioPortal and the analysis and interpretation of data was described in the Methods section (See supplementary files).

Ethics approval and consent to participate

No ethical approval was achieved since only publicly available data and materials were used in this study.

\section{Consent for publication}

Not applicable.

\section{Competing interests}

The authors declare that they have no competing interests.

\section{Author details}

${ }^{1}$ Department of Medical Ultrasound, Division of Interventional Ultrasound, The First Affiliated Hospital, Sun Yat-sen University, No. 58 Zhongshan 2nd Road, Guangzhou 510080, China. ${ }^{2}$ Department of Clinical Research, State Key Laboratory of Oncology in South China, Collaborative Innovation Center for Cancer Medicine, Sun Yat-sen University Cancer Center, 510060 Guangzhou, China.

Received: 20 August 2020 Accepted: 29 October 2020

Published online: 09 December 2020

\section{References}

1. Pardoll D. The blockade of immune checkpoints in cancer immunotherapy. Nat Rev Cancer. 2012;12(4):252-64.

2. Cao J, Yan Q. Cancer epigenetics, tumor immunity, and immunotherapy. Trends Cancer. 2020;6(7):580-92.

3. Kopp L, Ray A, Denman C, Senyukov V, Somanchi S, Zhu S, et al. Decitabine has a biphasic effect on natural killer cell viability, phenotype, and function under proliferative conditions. Mol Immunol. 2013;54:296-301.

4. Wu H, Chen $Y$, Wang Z, Zhao Q, He M, Wang Y, et al. Alteration in TET1 as potential biomarker for immune checkpoint blockade in multiple cancers. J Immunother Cancer. 2019;7(1):264.

5. Wrangle J, Wang W, Koch A, Easwaran H, Mohammad $H$, Vendetti F, et al. Alterations of immune response of non-small cell lung Cancer with Azacytidine. Oncotarget. 2013;4(11):2067-79.

6. Zheng H, Zhao W, Yan C, Watson C, Massengill M, Xie M, et al. HDAC inhibitors enhance T-cell chemokine expression and augment response to PD-1 immunotherapy in lung adenocarcinoma. Clin Cancer Res. 2016;22(16): 4119-32.

7. Krivtsov A, Armstrong S. MLL translocations, histone modifications and leukaemia stem-cell development. Nat Rev Cancer. 2007;7(11):823-33.

8. Liedtke M, Cleary M. Therapeutic targeting of MLL. Blood. 2009;113(24): 6061-8.

9. Kandoth C, McLellan M, Vandin F, Ye K, Niu B, Lu C, et al. Mutational landscape and significance across 12 major cancer types. Nature. 2013; 502(7471):333-9.

10. Rao RC, Dou Y. Hijacked in cancer: the KMT2 (MLL) family of methyltransferases. Nat Rev Cancer. 2015;15(6):334-46.

11. Shen X, Zhao B. Efficacy of PD-1 or PD-L1 inhibitors and PD-L1 expression status in cancer: meta-analysis. Bmj. 2018;362:k3529. 
12. Davis AA, Patel VG. The role of PD-L1 expression as a predictive biomarker: an analysis of all US Food and Drug Administration (FDA) approvals of immune checkpoint inhibitors. J Immunother Cancer. 2019;7(1):278.

\section{Publisher's Note}

Springer Nature remains neutral with regard to jurisdictional claims in published maps and institutional affiliations.

Ready to submit your research? Choose BMC and benefit from:

- fast, convenient online submission

- thorough peer review by experienced researchers in your field

- rapid publication on acceptance

- support for research data, including large and complex data types

- gold Open Access which fosters wider collaboration and increased citations

- maximum visibility for your research: over $100 \mathrm{M}$ website views per year

At BMC, research is always in progress. 